\title{
Considerações Sobre Queixas Relacionadas ao Trabalho em Pacientes de uma Clínica-Escola
}

\section{Considerations on Work-Related Complaints of Patients in a School-Clinic}

\section{Consideraciones Sobre Quejas Relacionadas Al Trabajo En Pacientes De Una Clínica-Escuela}

\author{
Vanderléia de Lurdes Dal Castel Schlindwein* \\ Universidade Federal de Rondônia - UNIR, Porto Velho, Rondônia, Brasil
}

\author{
Alex Lustosa Silva** \\ Universidade Federal de Rondônia - UNIR, Porto Velho, Rondônia, Brasil \\ Dimitri Henriques Daldegan Bueno*** \\ Universidade Federal de São Paulo - UNFESP, São Paulo, São Paulo, Brasil \\ Paulo Rogério Morais**** \\ Universidade Federal de Rondônia - UNIR, Porto Velho, Rondônia, Brasil
}

\begin{abstract}
RESUMO
O presente artigo tem como objetivo caracterizar as queixas relacionadas ao trabalho de pacientes que realizaram acolhimento no Serviço de Psicologia Aplicada/SPA de uma universidade federal da região Amazônica, entre os anos de 2011 e 2014, de modo a identificar aqueles que apresentam queixas de sofrimento relacionados ao trabalho. O estudo caracteriza-se como do tipo quantitativo, transversal, com análise documental, e nele foram analisadas, a partir dos prontuários, as variáveis sexo, idade, renda, profissão e queixa inicial. Observou-se que dos 672 usuários acima de 18 anos, $503(74,5 \%)$ eram do sexo feminino e $172(25,5 \%)$ eram do sexo masculino, destes $94(13,9 \%)$ apresentaram queixas envolvendo o trabalho e $581(86,1 \%)$ relataram outras queixas. No grupo com queixas relacionadas ao trabalho, as mulheres apresentaram maior prevalência nos atendimentos, entretanto são os homens que discorrem mais sintomas físico-psicológicos. Diante do exposto, a proposta de uma Clínica do Trabalho no Serviço de Clínica-escola, é necessária e deve incluir uma linha de cuidados/escuta evidenciando a relação do sujeito com seu trabalho, já que nem sempre o sofrimento psíquico relacionado ao trabalho produz sintomas claros e podem ser reduzidos somente à dimensão dos aspectos subjetivos de cada paciente.
\end{abstract}

Palavras-chave: atendimento psicológico, clínica-escola, queixas do trabalho. 


\begin{abstract}
The present study aimed to characterize the work-related complaints of patients who received treatment at the Applied Psychology Service/SPA of a federal university in the Amazon region between 2011 and 2014 in order to identify those patients presenting complaints of suffering at work. The study is quantitative, cross-sectional with documentary analysis, and analyzed, from the medical records, the variables gender, age, income, profession and initial complaint. Among the 672 users over 18 years of age, $503(74.5 \%)$ were female and $172(25.5 \%)$ were male. Of these, $94(13.9 \%)$ presented complaints involving work and $581(86.1 \%)$ reported other complaints. In the group with complaints related to work, women presented a higher prevalence in the consultations; however men showed more physicalpsychological symptoms. In view of the above, the proposal of a Work Clinic at the School-Clinic Service is necessary and should include care/listening showing the relation of the subject to his/her work, since not always the psychic suffering related to work produces clear symptoms and can be reduced only to the dimension of the subjective aspects of each patient.
\end{abstract}

Keywords: psychological care, school-clinic, complaints about the job.

\title{
RESUMEN
}

El presente artículo tiene como objetivo caracterizar las quejas relacionadas al trabajo de pacientes que realizaron acogida en el Servicio de Psicología Aplicada/SPA de una universidad federal de la región Amazónica, entre los años de 2011 y 2014, de modo a identificar aquellos que presentan quejas de sufrimiento relacionados al trabajo. El estudio se caracteriza como del tipo cuantitativo, transversal, con análisis documental, y en él se analizaron, a partir de los prontuarios, las variables sexo, edad, renta, profesión y queja inicial. Se observó qué de los 672 usuarios con más de 18 años, 503 (el $74,5 \%$ ) eran del sexo femenino y 172 (el 25,5\%) eran del sexo masculino, de estos 94 (el 13,9\%) presentaron quejas involucrando el trabajo y 581 (el $86,1 \%$ ) relataron otras quejas. En el grupo con quejas relacionadas al trabajo, las mujeres presentaron más prevalencia en las atenciones, sin embargo, son los hombres que discurren más síntomas físico-psicológicos. Delante de lo expuesto, la propuesta de una Clínica del Trabajo en el Servicio de Clínica-escuela, es necesaria y debe incluir una línea de cuidados/escucha evidenciando la relación del sujeto con su labor, ya que no siempre el sufrimiento psíquico relacionado al trabajo produce síntomas claros y pueden ser reducidos solamente a la dimensión de los aspectos subjetivos de cada paciente.

Palabras clave: atención psicológica, clínica-escuela, quejas de trabajo.

\section{I ntrodução}

Como reflexo das subjetivações, advindas da configuração atual do mundo socioeconômico, o trabalho passou a ser compreendido não apenas como fonte de renda material, mas, também, pode igualmente ocupar lugar central na construção da identidade e na realização pessoal, possibilitando vivências de sofrimento ancoradas nas experiências objetivas e subjetivas do trabalho. Para Dejours e Bègue (2010, p. 41), “(...) trabalhar não é apenas produzir, é também produzir-se a si mesmos". A Psicodinâmica do Trabalho/PDT 
compreende que não há neutralidade do trabalho em relação à identidade e à saúde mental. A relação com o trabalho pode gerar o melhor, mas pode da mesma forma levar à desestabilização, à crise de identidade ${ }^{1}$ e à descompensação psicopatológica. Os autores observam a dupla polaridade do trabalho: ele é tanto fonte de desgaste e sofrimento quanto meio de sublimação ${ }^{2}$ prazer $\mathrm{e}$ realizações.

Compreende-se que o trabalho é um dos grandes alicerces da constituição do sujeito e da sua rede de significados, uma vez que processos como reconhecimento, gratificação e mobilização da inteligência, ainda que estejam relacionados à realização do trabalho, também estão similarmente vinculados à constituição da identidade e da subjetividade (Lancman \& Sznelwar, 2011).

Esta relação vem sendo repensada nos serviços de Psicologia no Brasil, haja vista que professores e alunos de Psicologia estão sendo desafiados a compreender, diagnosticar as queixas frequentes de trabalhadores com sintomas e patologias psíquicas relacionadas ao trabalho. Para Merlo, Bottega e Perez (2014) é preciso construir uma Clínica do Trabalho que possa acolher as demandas advindas do contexto do trabalho, para isso a formação e a experiência são fundamentais para uma melhor intervenção baseada em dispositivos terapêuticos eficazes.

Nessa perspectiva, o presente estudo fomenta discussões sobre a necessidade de uma escuta diferencial para as queixas relacionadas ao trabalho nos serviços de acolhimento nos Serviços de Psicologia Aplicada (SPA) dos cursos de Psicologia no país. O interesse pela pesquisa surgiu a partir da implantação do primeiro estágio curricular em Clínica do Trabalho ${ }^{3}$ numa clínica-escola de uma universidade federal, ofertado a partir do ano de 2014.

De acordo com o projeto político-pedagógico do curso de graduação em Psicologia da instituição, o SPA foi fundado no ano de 1993 e configura-se como uma clínica-escola, já que objetiva oferecer atendimento gratuito a toda a comunidade, associado a possibilidade de realizar atividades de ensino, pesquisa e extensão com os alunos que compõem a efetivação do serviço.

Ao longo desses anos de funcionamento o SPA se consolidou como referência em acompanhamento psicológico nesta região, oferecendo atendimentos nas modalidades individuais e em grupo (Lima et al., 2010). Além da procura espontânea, diversos pacientes vêm ao serviço por meio de encaminhamento médico ou escolar, geralmente provenientes de instituições como os Centros de Atenção Psicossocial (CAPS) ou da rede pública de ensino. A partir de 2014 o SPA passou a integrar os encaminhamentos do Subsistema Integrado de Atenção à Saúde do Servidor Público Federal (SIASS) do estado.

Para Mendes e Araújo (2012), a Clínica Psicodinâmica do Trabalho é um modo de desvelar as mediações que ocorrem entre o sujeito e o 
real do trabalho. Compreende-se que o real pode ser desvelado pela escuta do clínico quanto à fala do trabalhador, o que, por sua vez, pode dar visibilidade às situações vivenciadas no trabalho. Por ser uma clínica, a palavra, enquanto linguagem e forma de comunicação, é essencial para que as experiências no trabalho sejam entendidas, percebidas e ressignificadas e o sujeito tenha a possibilidade de se reposicionar diante da objetividade/subjetividade mobilizada nas relações de trabalho.

A proposta de um estágio em conformidade com os pressupostos metodológicos da psicodinâmica do trabalho no SPA contribui para a constituição da clínica-escola enquanto espaço público e dispositivo capaz de viabilizar ao sujeito as estratégias criativas para compreensão e enfrentamento do sofrimento no trabalho (Oliveira, Silva, Lopes, Prado, Falcão \& Schlindwein, 2014; Merlo et al., 2014, Mendes, 2015). Assim como, pode oferecer um espaço que possibilite ao trabalhador uma escuta compreensiva e acolhedora que 0 questione como se sente no seu trabalho. Experiências dos serviços que atendem à saúde mental relacionada ao trabalho têm mostrado que basta uma simples questão de como se sente no trabalho é suficiente para que o trabalhador possa falar da sua atividade, desencadeando crises de choro e de ansiedade (Merlo, 2014; Ghizoni et al., 2014).

Nesse sentido, o presente estudo reitera a importância, para o curso de Psicologia, de conhecer as características ocupacionais dos sujeitos que buscam o Serviço de Psicologia da instituição e de possibilitar a identificação, já no processo de acolhimento, das queixas relacionadas ao trabalho, de modo que possam ser encaminhados para o atendimento em Clínica do Trabalho.

\section{Método}

A abordagem metodológica utilizada foi de caráter quantitativo com delineamento transversal, com análise documental e de cunho descritivo (Gil, 2002), e teve como objetivo descrever o perfil ocupacional e as queixas descritas nos prontuários do Serviço de Psicologia Aplicada da Universidade Federal de Rondônia (SPA/UNIR) que tinham relação com o trabalho, no período de 2011 a 2014.

Os dados foram coletados nos arquivos com as fichas de triagem do SPA/UNIR. A partir disso, realizou-se uma investigação e sistematização das informações contidas nos itens referentes à identificação do usuário. Os dados sociodemográficos contidos no documento foram preenchidos pela técnica responsável pela secretaria do SPA, enquanto as descrições das queixas foram preenchidas por estagiários de Psicologia após o procedimento de 
triagem dos pacientes, sempre sob a supervisão do professor orientador.

Os critérios de inclusão adotados para a seleção das fichas de triagens foram 0 ano em que o usuário realizou a triagem (entre 2011 e 2014) e a idade do usuário (igual ou superior a 18 anos). Após a seleção das fichas, os usuários foram dispostos em dois grupos, de acordo com base a queixa principal identificada em sua ficha ("Pessoas com queixas relacionadas ao trabalho" e "Pessoas com outras queixas"). Para a caracterização dos grupos foram organizadas em um banco de dados informações sobre o sexo, a idade, a escolaridade, a renda, a ocupação profissional e a síntese da queixa do momento da triagem. As queixas que tinham relação com o trabalho foram identificadas e classificadas de acordo com duas dimensões: aspectos psicológicos e aspectos físico-psicológicos.

Para fins de análise, foi criada uma categorização da ocupação profissional em grupos. Tomou-se como base a Classificação Brasileira de Ocupações, que define o conceito de ocupação como (Brasil, 2010, p. 07) “(...) agregação de empregos ou situações de trabalho similares quanto às atividades realizadas. $O$ título ocupacional, em uma classificação, surge da agregação de situações similares de emprego". Foram feitas as devidas adaptações em razão da demanda apresentada nos documentos pesquisados. Tal categorização resultou no agrupamento em 15 áreas: administrativa, do agronegócio, dos aposentados, das artes, dos autônomos, do comércio, dos desempregados, de pessoas do lar, da Educação, dos estudantes, dos profissionais liberais (advogados, engenheiros civil e elétrico, jornalistas, técnicos, etc.), da Saúde, da Segurança, do Serviço Público e dos serviços. Esse agrupamento não só facilitou a compreensão de algumas variáveis de cada profissão, mas da mesma forma permitiu a comparação de indicadores como sexo, ano de atendimento no SPA, idade e renda, e ainda a comparação entre o grupo que apresentou queixas de trabalho e o dos que apresentaram as outras queixas.

As análises estatísticas foram realizadas com o auxílio do software Statistical Package for the Social Sciences, versão 20.0 (SPSS-20.0). As características sociodemográficas foram identificadas por meio dos valores absolutos e suas porcentagens. Para verificar a existência de diferenças significativas em variáveis nominais foi utilizado o teste do Qui-quadrado. O teste T de Student foi utilizado para as comparações de variáveis paramétricas com distribuição simétrica, e para os dados com distribuição assimétrica foi utilizado o teste $U$ de Mann-Whitney. Em todos os casos, o nível de significância foi de 5\%. (Morais, 2007). Como procedimento ético, a pesquisa foi autorizada pela Coordenadoria do SPA da Universidade e aprovado pelo Comitê de Ética em Pesquisa da instituição CAAE: 48093015.7.0000.5300. 


\section{Resultados e Discussão}

No SPA, no período de 2011 a 2014 houve 675 solicitações de atendimentos de adultos (18 anos ou mais), deste universo 503 $(74,5 \%)$ eram do sexo feminino e $172(25,5 \%)$ eram do sexo masculino, predominando o atendimento às mulheres. Do total de acolhimento realizado pelos estagiários de Psicologia, 94 (13,9\%) apresentaram queixas relacionadas ao trabalho e $581(86,1 \%)$ apresentam outras queixas. A tabela 1 monstra a percentagem, por sexo, do grupo de pessoas que apresentaram queixas relacionadas ao trabalho. Após tratamento dos dados, o teste do Qui-quadrado detectou que havia mais mulheres que passaram no processo de triagem tanto no grupo de pessoas com queixas relacionadas ao trabalho quanto no grupo de pessoas com outras queixas $\left(x^{2}=6,12\right.$; $g \mid=1 ; p=0,013$ e $x^{2}=162,21 ; g l=1 ; p<0,001$, respectivamente). Mas, na comparação entre os dois grupos (homens e mulheres) com queixas relacionadas ao trabalho revelou que, embora as mulheres apresentam maior prevalência nos atendimentos, são os homens que apresentam mais descrições de sintomas físico-psicológicos. $\left(x^{2}=30,42 ; g l=1 ; p<0,001\right)$.

Tabela 1.

Frequência e porcentagem de sexo no grupo que apresentaram queixas relacionadas ao trabalho e do grupo com as demais queixas.

\begin{tabular}{|c|c|c|c|c|}
\hline \multirow{2}{*}{ Sexo } & \multicolumn{2}{|c|}{$\begin{array}{c}\text { Pessoas com } \\
\text { queixas } \\
\text { relacionadas ao } \\
\text { trabalho }\end{array}$} & \multicolumn{2}{|c|}{$\begin{array}{l}\text { Pessoas com } \\
\text { outras queixas }\end{array}$} \\
\hline & 35 & $37,2 \%$ & 137 & $23,6 \%$ \\
\hline Feminino & 59 & $62,8 \%$ & 444 & $76,4 \%$ \\
\hline Total & 94 & $100 \%$ & 581 & $100 \%$ \\
\hline
\end{tabular}

O predomínio de usuários do sexo feminino entre os que procuraram atendimento psicológico no SPA/UNIR está em consonância com oS achados de outros estudos que descrevem a clientela de clínicasescola. Os dados do presente estudo corroboram os de pesquisas já realizadas em outros serviços de Psicologia, indicando a prevalência do feminino nos atendimentos de clínicas-escolas, como descrito nas pesquisas de Ancona-Lopez (1983), Campezatto e Nunes (2007), J usten et al. (2010), Santos, Freitas e Rodrigues (2015).

Sobre a prevalência da população feminina entre os usuários de serviços de Psicologia, Lennon (1995), identificou que as mulheres 
apresentavam consideravelmente mais sintomas de angústia psicológica e desordens depressivas do que os homens. Uma explicação para isto estaria na sobrecarga de trabalho da mulher com outras jornadas de trabalho - como atividades domésticas e cuidados com os filhos. Araújo, Pinho e Almeida (2005) e Vidal e Neto (2009) descrevem a prevalência de transtornos mentais e comportamentais entre mulheres e exploram os fatores relacionados às características sociodemográficas e aspectos do trabalho doméstico potencialmente associados aos transtornos mentais. Observaram que a mulher, mesmo após sua inserção no mercado de trabalho, permanece como a principal responsável por planejar e executar as atividades domésticas, sem receber ajuda substancial dos companheiros e filhos, e que o auxílio nas tarefas, quando disponível, é proveniente de outra mulher. Os estudos sugerem que aspectos relacionados ao trabalho doméstico podem estar associados a repercussões negativas na saúde mental das mulheres.

Os dados apontam que, entre os dois grupos, há mais descrições de queixas relacionadas ao trabalho nos prontuários dos homens do que nos prontuários do grupo feminino. Sobre isso, Macedo, Silva, Giaretta, Ribas e Druck (2010), num estudo realizado com usuários masculinos adultos em uma clínica-escola de uma universidade privada na Região Metropolitana de Porto Alegre no período de janeiro de 2006 a julho de 2009, concluíram que os homens não apenas têm buscado ajuda por encaminhamento de outras fontes, como também têm ido espontaneamente aos serviços de saúde. Os autores destacam que tal constatação resulta da maior atenção que vem sendo dispensada à saúde masculina (preocupação explicitada pelo Ministério da Saúde em relação a um cuidado integral à saúde masculina); porém é pertinente considerar que a procura espontânea pode estar atrelada à intensificação de sofrimento psíquico.

Na tabela 2, o teste $U$ de mann-whitney detectou que o grupo de pessoas com queixa relacionada ao trabalho não difere do das pessoas com outras queixas na variável idade $(U=23071 ; p=0,076)$. $\mathrm{O}$ mesmo teste demonstrou que as pessoas com queixa relacionada ao trabalho apresentam uma renda superior quando comparadas com o grupo de pessoas com outras queixas $(U=19735 ;=0,042)$. Podese observar que a população que procura o SPA/UNIR é constituída de trabalhadores com renda na faixa de 800 a 2.400 reais. Tal dado não está em consenso com os resultados encontrados em pesquisas acerca da descrição da população atendida em clínicas-escola: contudo, segundo esses estudos, a maior parte da clientela da clínica se constitui de pessoas menos favorecidas economicamente, com rendas inferiores a 1.000 reais, como indicam as pesquisas de Ancona-Lopez (1983), Carvalho e Telles (2001), Campezatto e Nunes (2007), Macedo et al. (2010), Justen et al. (2010) e outros. 
Tabela 2

Mediana e intervalo interquartil da idade (anos) e renda (R\$) nos grupos.

\begin{tabular}{|c|c|c|c|c|}
\hline Variável & \multicolumn{2}{|c|}{$\begin{array}{c}\text { Pessoas com queixas } \\
\text { relacionadas ao } \\
\text { trabalho }\end{array}$} & \multicolumn{2}{|c|}{$\begin{array}{c}\text { Pessoas com outras } \\
\text { queixas } \\
\text { Md (Q1-Q3) }\end{array}$} \\
\hline Idade & 30 & 25,$75 ; 38,50$ & 33 & $25 ; 43$ \\
\hline Renda & 1.500 & $1.000 ; 2.400$ & 1.200 & $800 ; 2000$ \\
\hline
\end{tabular}

Ao se compararem as categorias de profissões entre os grupos (tabela 3), o teste do Qui-quadrado demonstrou que há mais pessoas com queixa relacionada ao trabalho nas categorias: agronegócio $\left(x^{2}=16,90 ; g \mid=1 ; p<0,001\right)$, educação $\quad\left(x^{2}=5,29 ; g l=1 ; p=0,021\right)$, saúde $\quad\left(x^{2}=27,92 ; g l=1 ; p<0,001\right) \quad$ e segurança $\left(x^{2}=59,55 ; g l=1 ; p=0,001\right)$. Já no grupo de pessoas que apresentaram queixas não relacionadas ao trabalho, o mesmo teste demonstrou maior incidência de atendimentos nas especificações ocupacionais de: aposentado $\left(x^{2}=16,20 ; g l=1 ; p<0,001\right)$, artes $\left(x^{2}=6,40 ; g \mid=1 ; p=0,011\right)$, do lar $\left(x^{2}=24,02 ; g l=1 ; p<0,001\right)$, estudante $\left(x^{2}=16,19 ; g l=1 ; p<0,001\right)$ e profissional liberal $\left(x^{2}=16,20 ; g l=1 ; p<0,001\right)$.

$\mathrm{Na}$ análise da categoria das profissões (tabela 3 ) no atendimento do SPA/UNIR, observa-se que no grupo das queixas relacionadas ao trabalho, nos setores do comércio as queixas são observadas em $12,8 \%$ dos prontuários; nos da segurança e da educação, em 11,7\%; no da saúde, 10,6\%; nos dos estudantes, dos trabalhadores do lar e no do serviço público, cerca de $6,4 \%$ manifestaram queixas sobre trabalho desenvolvido. Em outras ocupações observaram-se índices menores, como: no setor administrativo, 4,3\%; no dos autônomos e no agronegócio, 3,2\%; e no dos desempregados, $1,1 \%$.

Em outros estudos realizados em clínicas-escola observam-se percentuais maiores aos encontrados para a categoria dos estudantes, como de Campezatto e Nunes (2007) e Justen et al. (2010). Nestes estudos, em média quase a metade da clientela atendida é de estudantes. Sendo assim, o estudo realizado nos prontuários do serviço de Psicologia da universidade não houve predomínio de estudantes, o que demonstra que o SPA vem 
constituindo-se como um espaço de referência no atendimento à diversas áreas do setor público e privado da comunidade.

Tabela 3

Frequência e porcentagem de categorias de profissões nos grupos.

\section{Categoria de profissão}

\section{Pessoas com queixas relacionadas ao trabalho}

Pessoas com outras queixas

\begin{tabular}{|c|c|c|c|c|}
\hline Comércio & 12 & $12,8 \%$ & 67 & $11,5 \%$ \\
\hline Segurança & 11 & $11,7 \%$ & 13 & $2,2 \%$ \\
\hline Saúde & 10 & $10,6 \%$ & 26 & $4,5 \%$ \\
\hline Estudante & 6 & $6,4 \%$ & 80 & $13,8 \%$ \\
\hline Educação & 11 & $11,7 \%$ & 41 & $7,1 \%$ \\
\hline Dolar & 6 & $6,4 \%$ & 80 & $13,8 \%$ \\
\hline Serviço público* & 6 & $6,4 \%$ & 27 & $4,6 \%$ \\
\hline Administrativo & 4 & $4,3 \%$ & 28 & $4,8 \%$ \\
\hline Autônomo & 3 & $3,2 \%$ & 26 & $4,5 \%$ \\
\hline Agronegócio & 3 & $3,2 \%$ & 4 & $0,7 \%$ \\
\hline Desempregados & 1 & $1,1 \%$ & 4 & $0,7 \%$ \\
\hline Aposentados & 0 & $0 \%$ & 11 & $1,9 \%$ \\
\hline Artes & 0 & $0 \%$ & 5 & $0,9 \%$ \\
\hline Profissional liberal & 0 & $0 \%$ & 11 & $1,9 \%$ \\
\hline \multicolumn{5}{|c|}{$\begin{array}{l}\text { Fonte: Elaborada pelos autores a partir do banco de dados dos prontuários } \\
\text { do Serviço de Psicologia Aplicada/SPA/UNIR }\end{array}$} \\
\hline
\end{tabular}

Um estudo realizado por Campezatto e Nunes (2007) em que se utilizaram os métodos quantitativo, descritivo e de levantamento, buscou conhecer a população que frequentou as 23 clínicas-escola existentes no Estado no Rio Grande do Sul no ano de 2004, incluindo nível socioeconômico e ocupação. O estudo identificou o predomínio do sexo feminino, com $59,73 \%$, sendo o maior perfil ocupacional o de estudantes, com $42 \%$. Os números incluem adultos e crianças. Trabalhadores não manuais de baixo padrão, como auxiliares de escritório e trabalhadores de serviços gerais, totalizaram 9,36\%. 
Outro estudo - este realizado por Justen et al. (2010) com 2.953 clientes de idades variadas que passaram por triagem no Centro de Psicologia Aplicada (CPA) da Universidade Paranaense no período de 1993 a 2006 - mostrou que cerca de $23 \%$ da população atendida no serviço correspondem a estudantes, vindo a seguir o grupo de empregados (carteira assinada ou serviço público), com 18,4\%, o dos que não possuem ocupação formal (do lar), com $11,6 \%$, e o de autônomos, com $8,3 \%$. Os resultados encontrados indicam a necessidade de padronização dos instrumentos de triagem, considerando-se a amplitude das variáveis pesquisadas. O achado fortalece a perspectiva de aprimoramento dos modelos de atendimento na clínica-escola.

Um estudo realizado em uma universidade privada de Porto Alegre com a utilização do método quantitativo do tipo transversal, contemplou 208 fichas de triagem de clientes do sexo masculino e de idade superior a 18 anos que buscaram a clínica-escola no período de julho de 2006 a janeiro de 2009. Os resultados mostraram que a faixa etária de maior concentração da amostra na pesquisa foi a de 20 a 26 anos, com 22,60\%. Mostraram que 55,8\% dos sujeitos estavam inseridos em contexto laboral, como empregados ou autônomos, que $20,7 \%$ eram estudantes e que $14,9 \%$ não trabalhavam (Macedo et al., 2010).

O teste do Qui-quadrado (tabela 4) detectou maior incidência de queixas físico-psicológicas relacionadas ao trabalho $\left(x^{2}=12,29 ; g l=1 ; p<0,001\right)$ nos atendimentos de acolhimento realizadas no SPA/UNIR. As queixas psicológicas abrangeram descrições como sentimento de medo, perseguição no trabalho, dificuldades nas relações de trabalho, crises de choro, angústia, ansiedade, desânimo, pressões, cobranças, tentativas de suicídio, irritação, agressividade, tristeza, frustração, pensamentos suicidas, baixa autoestima, sentimento de bloqueio emocional, desânimo, falta de desmotivação, conflitos da área profissional, perseguição no trabalho, conflitos, choro, sobrecarga de trabalho, isolamento, sofrimento, timidez, bloqueio nas relações interpessoais. Por sua vez, as queixas de caráter físico-psicológico caracterizavam-se por estresse, alterações de sono, cansaço físico, dificuldades na alimentação; dores de cabeça, incontinência urinária, queda de cabelo, manchas na pele, dores pelo corpo, falta de ar, fibromialgia, fadiga crônica, tremores, taquicardia e esgotamento físico. Não se encontrou nenhum outro estudo com este recorte, entretanto é possível afirmar que queixas semelhantes são encontradas em estudos que avaliam a demanda geral das clínicas-escolas no Brasil, como de Romaro e Capitão (2003), Romaro e Oliveira (2008) e Santos et al. (2015). 
Tabela 4

Frequência e porcentagem das categorias de queixas.

\begin{tabular}{lc}
\hline $\begin{array}{l}\text { Categoria das } \\
\text { queixas }\end{array}$ & Pessoas com queixas relacionadas ao \\
trabalho
\end{tabular}

Psicológicas

Físico-psicológicas

Total

94
$68,1 \%$

$31,9 \%$

$100 \%$

Fonte: Elaborada pelos autores a partir do banco de dados dos prontuários do Serviço de Psicologia Aplicada/SPA/UNIR.

Observa-se que, entre os indivíduos que apresentam queixas relacionadas ao trabalho há maior incidência de sintomas psicológicos. Os sintomas psíquicos relacionados ao trabalho se manifestam por todo o corpo. Os mesmos sintomas são descritos por Merlo (2014) na prática dos atendimentos do Ambulatório de Doenças do Trabalho do Hospital de Clínicas de Porto Alegre (ADT-HCPA). O autor aponta como os principais sintomas encontrados: insônia, uso regular de bebidas alcoólicas, de maconha (cannabis) ou de tranquilizantes; como sintomas físicos: astenia, dores abdominais, dores nas costas, dores musculares, dores articulares, distúrbios do sono, distúrbios do apetite, etc.; como sintomas emocionais: irritabilidade aumentada, angústia, ansiedade, excitação, tristeza, choro frequente, sentimentos de mal-estar indefinidos, etc.; como sintomas intelectuais; distúrbios de concentração de memória, dificuldades para tomar iniciativas ou decisões, etc.; e como sintomas comportamentais: modificação de hábitos alimentares, isolamento social e dificuldades para cooperar, etc.

Diante do exposto, a proposta de uma Clínica do Trabalho no Serviço de Psicologia Aplicada, deve incluir uma linha de cuidados/escuta evidenciando a relação do sujeito com seu trabalho. Como dispositivo analítico o estagiário apreende a desenvolver uma postura mais compreensiva e investigativa quando se trata de sintomas e sinais de que aspectos do trabalho tem contribuído para uma desestabilização dos afetos, sentimentos e percepções. O sofrimento advindo do trabalho/ou a falta dele é a expressão do significado psicológico, subjetivo e presentifica a ação humana no mundo. Para Araújo (2013) "O trabalho não é neutro para o sujeito. Ele se engaja no corpo e no afeto" (p.91). 
A Clínica do Trabalho pode constituir-se como um espaço de escuta analítica das expressões psicológicas do sofrimento no trabalho, busca intervir nas expressões, sentimentos e desafetos com os colegas, frente a organização do trabalho. O psicológico é entendido como disposições individuais, a expectativas, a jeitos de ser e a ritmos individuais. Remete a diferenças do que a aspectos comuns entre as pessoas. Diz respeito a como internalizamos particularidades nos modos de ser na sociedade (crenças/valores/regras, mitos e padrões de comportamento) (Sato, 2003, p.70).

Finalmente, o estudo aponta à possibilidade de ampliação dos serviços nas Clínicas-escolas para uma escuta qualificada nos casos em que as queixas psicológicas estejam relacionadas a fatores do trabalho. Esta proposta deve auxiliar os sujeitos a promover a irrupção daquilo que se encontra bloqueado, levando à criação de novas estratégias, aciona-se neles a capacidade de se transformar, irrompendo novos significados, acolhendo e tirando os trabalhadores do lugar solitário em que são colocados.

\section{Considerações finais}

Com base nos achados do presente estudo, destaca-se que os serviços prestados por uma clínica-escola em uma instituição pública de ensino superior localizada na Região Norte do País contribuem não apenas para a formação acadêmica dos estudantes de Psicologia, mas também para o papel desta na atenção à saúde do trabalhador e da trabalhadora no Estado de Rondônia.

Os dados apresentados nessa clínica-escola ao longo de quatro anos demonstraram que as queixas relacionadas ao trabalho representam $13,9 \%$ do total de triagens realizadas pelo serviço, e nestas, as mulheres comparecem com maior frequência no número de prontuários, com $62,8 \%$, ficando os homens com 37,2\%. Nesse sentido, o estudo realizado ajuda a reafirmar que a clínica-escola é um espaço que vem contribuir com a Política Nacional da Saúde do Trabalhador e da Trabalhadora/PNSST, investigando e realizando o acompanhamento dos trabalhadores e trabalhadoras nos casos de sofrimento e/o adoecimento psíquico relacionados ao trabalho, bem como construir um espaço de acolhimento e escuta das queixas relacionadas ao trabalho.

A análise dos prontuários permite sinalizar aos estagiários e supervisores que nem sempre o sofrimento psíquico relacionado ao trabalho produz sintomas claros, que por vezes os sofrimentos são inespecíficos e podem ser associados somente à dimensão da história de vida do paciente. Neste contexto, são necessários novos estudos, particularmente com delineamentos que permitam 0 acompanhamento longitudinal nos prontuários de atendimento 
clínico, para esclarecer com maior detalhamento as possíveis relações entre as queixas, os sintomas e as diferentes dimensões relativas às atividades laborais.

Outra dimensão que o estudo possibilita reforçar é a importância de se fortalecer a pesquisa com foco nas queixas relacionadas ao trabalho em serviços oferecidos pelas clínicas-escola de Psicologia, como forma de aprimorar a formação do psicólogo e identificar possíveis meios de intervenção nos quadros de sofrimento psíquico produzido ou agravado pelo trabalho. Para Merlo (2014, p. 26), a primeira atitude que no atendimento tem um efeito terapêutico imediato é o paciente/trabalhador saber que não está mais só nesta situação, logo isto o retira do isolamento e da solidão. Entende o autor que é preciso fazer uma escuta compreensiva e acolhedora, de modo a levar o trabalhador a pensar na relação entre o sofrimento e o seu trabalho.

No que diz respeito a esta pesquisa, ainda é necessário destacar que a partir de 2014 iniciaram-se no SPA-UNIR os atendimentos em Clínica Psicodinâmica do Trabalho (Oliveira, Silva, Lopes, Prado, Falcão \& Schlindwein, 2014), o que pressupõe a possibilidade de realizar novos estudos, tendo em vista que muitos trabalhadores e trabalhadoras com queixas do trabalho, no momento do acolhimento, serão encaminhados para este atendimento. Para Marturano, Silvares e Oliveira (2014), é na função de capacitação de multiplicadores que o serviço-escola cumpre o seu papel, pois é com esta capacitação que a comunidade se apropria de um conhecimento já então parcialmente transposto para práticas que atendem a determinadas demandas.

Do ponto de vista da saúde mental, destaca-se que, é importante que no processo de formação dos futuros psicólogos, a partir dos estágios realizados nas clínicas-escola, possam desenvolver habilidades para reconhecer, compreender e tratar a demanda dos casos de sofrimento psíquico relacionado ao trabalho, os quais muitas vezes chegam disfarçados nas entrelinhas das queixas e sintomas.

\section{Referências}

Ancona-Lopez, M. A. (1983). Considerações sobre o atendimento fornecido por clínicas-escola de psicologia. Arquivos Brasileiros de Psicologia, 35(2), 123-135. Recuperado de http: // bibliotecadigital.fgv.br/ojs/index.php/abp/article/view/18 922

Araújo, T. M, Pinho, O. S., \& Almeida, M. M. G. (2005). Prevalência de transtornos mentais comuns em mulheres e sua relação com as características sociodemográficas e o trabalho doméstico Revista Brasileira de Saúde Materno Infantil. 5(3), 337-48. doi: http://dx.doi.org/10.1590/S1519-38292005000300010 
Araújo, L. K. R. (2013). Clínica do trabalho. In F. O. Vieira, A. M. Mendes, \& Á. R. C. Merlo, (Orgs.), Dicionário crítico de gestão e Psicodinâmica do Trabalho (pp. 89-92). Curitiba: Juruá.

Brasil. (2010). Classificação Brasileira de Ocupações: CBO. 3a ed. Brasília: MTE, SPPE. Recuperado de http:// wp.ufpel. edu.br/observatoriosocial/files/2014/09/CBOLivro-2.pdf

Campezatto, P. V. M., \& Nunes, M. L. T. (2007). Caracterização da clientela das clínicas-escola de cursos de Psicologia da região metropolitana de Porto Alegre. Psicologia: reflexão e crítica, 20(3), 376-388. doi: http://dx.doi.org/10.1590/S010279722007000300005

Carvalho, M. J. D. C., \& Telles, S. R. D. A. (2001). Considerações sobre queixas de pacientes em triagem de clínica-escola. Psikhê, 6(1), 7-14.

Dejours, C., \& Bègue, F. (2010). Suicídio e trabalho: o que fazer? Brasília: Paralelo 15.

Elias, L. C. S, Marturano, E. M., \& Motta-Oliveira, A. M. (2013). Teoria, pesquisa e prática em serviço-escola de psicologia. Saúde \& Transformação, 4(3), 121-129. Recuperado de http://incubadora. periodicos. ufsc. br/index. php/saudeetransfor macao/article/view/ 1978

Gelso, C. J. (2006). On the making of a scientist-practitioner: A theory of research training in professional psychology. Training and Education in Professional Psychology, S(1), 3-16. doi: http://dx.doi.org/10.1037/1931-3918.S.1.3

Gil, A. C. (2002). Como elaborar projetos de pesquisa. São Paulo: Ed. Atlas.

Ghizoni, L. D., Medeiros, S. N., Carvalho, G. M., Moraes, R. D., Santana, P. M., Magnus, C. N., Mendes, A. M. (2014). Clínica psicodinâmica do trabalho: a prática em diversos contextos de trabalho. Desafios: Revista Interdisciplinar da Universidade Federal do Tocantins, 1(1), 74-92. Recuperado de http: // revista. uft. edu. br/index. php/desafios/article/viewFile/79 6/74-92

Justen, A., Paltanin, E. S., Maroneze, G. S., Vissovatz, M. M., Prà, J . D., Feltrin, J., Silva, M. A., Mariussi, M. C., Pereira, R. S., \& Lima, O. M. P. (2010). Identificação da população atendida no centro de psicologia aplicada da Universidade Paranaense. Arquivos de Ciências da Saúde UNIPAR, 14(3), 197-209. Recuperado de http://revistas.unipar.br/saude/article/viewFile/3661/2374

Lancman, S., \& Sznelwar, L. I. (2011). Christophe Dejours: da psicopatologia à psicodinâmica do trabalho. Rio de J aneiro/Brasília: Fiocruz/Paralelo 15. 
Lennon, M. C. (1995). Work conditions as explanations for the relation between socioeconomic status, gender, and psychological disorders. Epidemiologic Reviews, 17(1), 120127.

Lima, V. A. A., Chagas, D. S. A., Marques, F. L. L., Vianna, J. J. B., Montes, M. C. M., Moura, M. M. C., \& Silva, A. A. M. (2010). Clínica-Escola: um olhar da Psicologia Institucional sobre o Serviço de Psicologia Aplicada da UNIR. Mnemosine, 6(2). Recuperado http://www.mnemosine.com.br/ojs/index. php/mnemosine/articl e/view/207

Macedo, M. M. K., Silva, F. C. F. D., Giaretta, D. G., Ribas, R. F., \& Druck, C. M. (2010). Atenção integral à saúde masculina: a busca por atendimento psicológico em uma clínica-escola. Psicologia: teoria e prática, 12(1), 154-170. Recuperado de http://pepsic. bvsalud.org/scielo. php?pid=S151636872010000100013\&script=sci_arttext

Marturano, E. M., Silvares, E. F. D. M., \& Oliveira, M. D. S. (2014). Serviços-escola de psicologia: seu lugar no circuito de permuta do conhecimento. Temas em Psicologia, 22(2), 457-470. doi: http://dx.doi. org/10.9788/TP2014.2-15.

Mendes, A. M., (2015). La escucha analítica del sufrimiento y el saber-hacer del clínico del trabajo. In A. Pujol \& M. I. Gutiérrez (Com.), Trabajo y subjetividade: indagaciones clínicas (pp 1122). Córdoba/Argentina: Licencia Creative Commons. Recuperado de http://www.simposiotas.com.ar/Libro TAS 2014.pdf

Mendes, A. M. (2007). Psicodinâmica do Trabalho: Teoria, Método e Pesquisas. Casa do psicólogo. São Paulo: Casa do Psicólogo.

Mendes, A. M., \& Araújo, L. K. R. (2012). Clínica psicodinâmica do trabalho - O sujeito em ação. Curitiba: Juruá.

Merlo, Á. R. C., Bottega, C. G., \& Perez, K. V. (2014). Atenção à saúde mental do trabalhador: sofrimento e transtornos psíquicos relacionados ao trabalho. Porto Alegre: Evangraf.

Merlo, Á. R. C. (2014). Sofrimento psíquico e atenção à saúde mental. In Á. R. C. Merlo, C. G. Bottega, \& K. V. Perez (Orgs.), Atenção à saúde mental do trabalhador: sofrimento e transtornos psíquicos relacionados ao trabalho (pp. 12-29). Porto Alegre/RGS: Evangraf.

Morais, P. R. (2007). Estatística para psicólogos que não gostam de números. Santo André/SP: EseTEC.

Oliveira, D. L. S, Silva, A. L., Lopes, F. R., Prado, F. B, Falcão, M. E. J., \& Schlindwein, V. L. D. C. (2014). Clínica do trabalho: relato de experiência em uma clínica-escola de Porto Velho - RO. In Anais do IV Congresso Brasileiro Psicologia: Ciência e Profissão - Fórum de Entidades Nacionais da Psicologia Brasileira. São 
Paulo, SP: Recuperado de http: //www.cienciaeprofissao.com. br/.

Romaro, R. A., \& Capitão, C. G. (2003). Caracterização da clientela da clínica-escola de psicologia da Universidade São Francisco. Revista Psicologia-Teoria e Prática, 5(1), 111-121. Recuperado de

http: // pepsic. bvsalud.org/scielo.php?script=sci_arttext\&pid=S1 516-36872003000100009\&lng=pt\&tlng=pt.

Romaro, R. A., \& Oliveira, P. E. C. L. (2008). I dentificação das queixas de adultos separados atendidos em uma clínica-escola de Psicologia. Psicologia: Ciência e Profissão, 28(4), 780-793. doi: http://dx.doi.org/10.1590/S1414-98932008000400010.

Santos, C. da S., Freitas, M. L. S., \& Rodrigues V. D. (2015). Perfil dos pacientes atendidos no setor de psicologia em uma clínicaescola. Revista Digital EFDeportes.com. Buenos Aires, Argentina. Recuperado de http: // www. efdeportes.com/efd202/perfil-dos-pacientes-nosetor-de-psicologia.htm

Sato, L. (2003). Subjetividade, saúde mental e LER. In R. C. Ruiz, L. Magalhães, L. Sato, M. Maeno, M. G. L. Hoefel, \& S. G. S. Mérola (Orgs.), Um mundo sem LER é possível (pp. 62-78). Montevidéu/Uruguai.

Vidal, R. D. Q. S., \& Neto, A. M. S. (2009). Trabalhadoras brasileiras: características socioeconômicas e ocupacionais e perfil de saúde, Brasil, 2003. Revista Brasileira de Saúde Ocupacional, 34(120), 115-127. doi: http://dx.doi.org/10.1590/S030376572009000200003

\section{Endereço para correspondência}

\section{Vanderléia de Lurdes Dal Castel Schlindwein}

Universidade Federal de Rondônia - UNIR

Campus - BR 364, Km 9,5, CEP 76801-059, Porto Velho - RO, Brasil

Endereço eletrônico: vdalcastel@gmail.com

\section{Alex Lustosa Silva}

Universidade Federal de Rondônia - UNIR

Campus - BR 364, Km 9,5, CEP 76801-059, Porto Velho - RO, Brasil

Endereço eletrônico: alexlustosasilva@gmail.com

\section{Dimitri Henriques Daldegan Bueno}

Universidade Federal de São Paulo - UNFESP

Rua Botucatu, 862, $1^{\circ}$. Andar, Vila Clementino, CEP 04023-062, São Paulo - SP, Brasil

Endereço eletrônico: dimitribueno@gmail.com

\section{Paulo Rogério Morais}

Universidade Federal de Rondônia - UNIR

Campus - BR 364, Km 9,5, CEP 76801-059, Porto Velho - RO, Brasil

Endereço eletrônico: moraispr@bol.com.br 
Recebido em: 29/10/2105

Reformulado em: 21/10/2016

Aceito em: 28/03/2017

\section{Notas}

* Professora da Pós-graduação em Psicologia (MAPSI) da Universidade Federal de Rondônia (UNIR). Porto Velho - RO - Brasil.

** Psicólogo, especialista em Ergonomia na Universidade Estadual de Londrina (UEL). Londrina - PR - Brasil.

*** Mestrando do Programa de Pós-graduação em Psicobiologia da Universidade Federal de São Paulo (UNFESP). São Paulo - SP - Brasil.

**** Professor da graduação em Psicologia da Universidade Federal de Rondônia (UNIR). Porto Velho - RO - Brasil.

${ }^{1}$ Etimologicamente, o termo identidade vem da palavra latina idem, significando o mesmo, a mesma. Do grego, é sinônimo de identificação e reconhecimento. Já na definição em português encontramos identidade como um conjunto de caracteres que fazem reconhecer um indivíduo.

${ }^{2}$ Sublimação, para a psicodinâmica do trabalho, desempenha papel de destaque na construção da identidade. Sublimar não é apenas mobilizar a nossa subjetividade; é ainda - independentemente de nossa vontade - engajar-nos na ação para o reconhecimento social de nosso trabalho por nossos pares e pela sociedade (Lancman \& Sznelwar, 2011).

3 A Clínica do Trabalho para Mendes (2015) privilegia a escuta analítica do sofrimento no trabalho, associado aos pressupostos teóricos da Psicanálise.

Este artigo de revista Estudos e Pesquisas em Psicologia é licenciado sob uma Licença Creative Commons Atribuição-Não Comercial 3.0 Não Adaptada. 